\title{
Final results of a phase 3 study of trebananib plus weekly paclitaxel in recurrent ovarian cancer (TRINOVA-1): Long-term survival, impact of

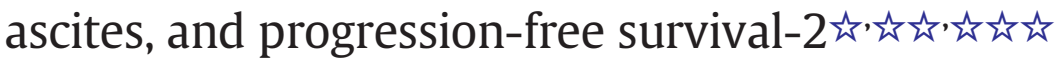

Bradley J. Monk ${ }^{\mathrm{a}, *}$, Andrés Poveda ${ }^{\mathrm{b}}$, Ignace Vergote ${ }^{\mathrm{c}}$, Francesco Raspagliesi ${ }^{\mathrm{d}}$, Keiichi Fujiwara ${ }^{\mathrm{e}}$, Duk-Soo Bae ${ }^{\mathrm{f}}$, Ana Oaknin ${ }^{\mathrm{g}}$, Isabelle Ray-Coquard ${ }^{\mathrm{h}}$, Diane M. Provencher ${ }^{\mathrm{i}}$, Beth Y. Karlan ${ }^{\mathrm{j}}$, Catherine Lhommé ${ }^{\mathrm{k}}$, Gary Richardson ${ }^{1}$, Dolores Gallardo Rincón ${ }^{\mathrm{m}}$, Robert L. Coleman ${ }^{\mathrm{n}}$, Christian Marth ${ }^{\mathrm{o}}$, Arija Brize ${ }^{\mathrm{p}}$, Michel Fabbro ${ }^{\mathrm{q}}$, Andrés Redondo ${ }^{\mathrm{r}}$, Aristotelis Bamias ${ }^{\mathrm{s}}$, Haijun Ma ${ }^{\mathrm{t}}$, Florian D. Vogl ${ }^{\mathrm{u}}$, Bruce A. Bach ${ }^{\mathrm{u}}$, Amit M. Oza ${ }^{\mathrm{v}}$

a Department of Obstetrics and Gynecology, University of Arizona Cancer Center at Dignity Health St. Joseph's Hospital and Medical Center, Phoenix, AZ, USA

b Area Clinica de Oncologia Ginecológica, Fundación Instituto Valenciano de Oncología, Valencia, Spain

c Department of Obstetrics and Gynecology, University Hospital Leuven, Leuven Cancer Institute, KU Leuven, European Union, Belgium

d Gynecologic Oncology Unit, Fondazione IRCCS, Istituto Nazionale per la Cura e lo Studio dei Tumori, Milano, Italy

e Department of Gynecologic Oncology, Saitama Medical University International Medical Center, Hidaka-Shi, Japan

${ }^{\mathrm{f}}$ Department of Obstetrics and Gynecology, Samsung Medical Center, Seoul, South Korea

${ }^{g}$ Vall d'Hebron University Hospital, Vall d'Hebron Institute of Oncology, Barcelona, Spain

${ }^{\mathrm{h}}$ Université Lyon-I, Centre Léon Bérard, Lyon, France

${ }^{i}$ Division of Gynecologic Oncology, Centre Hospitalier de l'Université de Montréal, Montreal, QC, Canada

${ }^{j}$ Women's Cancer Program at the Samuel Oschin Comprehensive Cancer Center, Cedars-Sinai Medical Center, Los Angeles, CA, USA

${ }^{\mathrm{k}}$ Department of Medicine, Institut Gustave Roussy, Villejuif, France

${ }^{1}$ Academic Haematology and Oncology, Cabrini Hospital, Malvern, VIC, Australia

m Subdirección de Medicina Interna, Instituto Nacional de Cancerologia, Mexico City, Mexico

n Department of Gynecologic Oncology and Reproductive Medicine, University of Texas MD Anderson Cancer Center, Houston, TX, USA

- Universitätsklinik für Gynäkologie und Geburtshilfe, Medizinische Universität Innsbruck, Innsbruck, Austria

${ }^{\mathrm{p}}$ Latvian Oncology Center, Riga Eastern Clinical University Hospital, Riga, Latvia

${ }^{\text {q }}$ Regional Cancer Institute Montpellier, Montpellier, France

${ }^{\mathrm{r}}$ Hospital Universitario La Paz Idi-Paz, Madrid, Spain

${ }^{s}$ Alexandra Hospital, Department of Clinical Therapeutics, National \& Kapodistrian University of Athens, Athens, Greece

${ }^{\mathrm{t}}$ Global Biostatistical Science, Amgen Inc., Thousand Oaks, CA, USA

u Global Development Oncology, Amgen Inc., Thousand Oaks, CA, USA

v Department of Medicine, Princess Margaret Hospital, University of Toronto, ON, Canada

\section{H I G H L I G H T S}

- Trebananib did not improve overall survival in the intent-to-treat population.

- Trebananib improved overall survival in patients with baseline ascites.

- Trebananib prolonged time to second disease progression.

\section{A R T I C L E I N F O}

\section{Article history:}

Received 6 May 2016

Received in revised form 12 July 2016

Accepted 23 July 2016

Available online $\mathrm{xxxx}$

\section{A B S T R A C T}

Purpose. Trebananib, a peptibody that blocks binding of angiopoietin-1 and -2 to Tie2, significantly prolonged progression-free survival (PFS) in patients with recurrent epithelial ovarian cancer in the phase 3 TRINOVA-1 study. We report overall survival (OS) in the intent-to-treat population and clinically relevant subgroups and time to second disease progression (PFS-2).

¿t Research support: This work was funded by Amgen Inc.

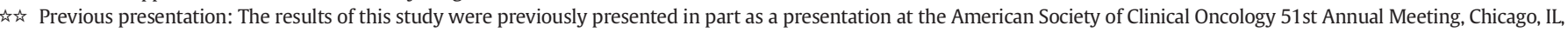
May 29-June 2, 2015.

论论论 Trial registration: ClinicalTrials.gov, NCT01204749.

* Corresponding author at: Division of Gynecologic Oncology, Department of Obstetrics and Gynecology, Creighton University School of Medicine at St. Joseph's Hospital and Medical Center, University of Arizona Cancer Center, Phoenix, AZ 85013, USA

E-mail address: bradley.monk@chw.edu (B.J. Monk). 
Keywords:

Trebananib

TRINOVA-1

Recurrent epithelial ovarian cancer

Overall survival

Ascites

Time to second disease progression
Patients and methods. Women with recurrent disease (platinum-free interval $<12$ months) were randomized to receive intravenous paclitaxel $80 \mathrm{mg} / \mathrm{m}^{2}$ ( 3 weeks on $/ 1$ week off) plus intravenous trebananib $15 \mathrm{mg} / \mathrm{kg}$ or placebo, weekly. OS in the intent-to-treat population was a key secondary endpoint. Exploratory analysis of PFS-2 was conducted according to guidance by the European Medicines Agency.

Results. Median OS was not significantly improved with trebananib compared with placebo (19.3 versus 18.3 months; HR, $0.95 ; 95 \% \mathrm{CI}, 0.81-1.11 ; P=0.52)$ in the intent-to-treat population $(\mathrm{n}=919)$. In subgroup analysis, trebananib improved median OS compared with placebo (14.5 versus 12.3 months; $\mathrm{HR}, 0.72$; $95 \% \mathrm{CI}$, $0.55-0.93 ; P=0.011)$ in patients with ascites at baseline $(\mathrm{n}=295)$. In the intent-to-treat population, trebananib significantly improved median PFS-2 compared with placebo (12.5 versus 10.9 months; $\mathrm{HR}, 0.85 ; 95 \% \mathrm{CI}$, $0.74-0.98 ; P=0.024)$. The incidence and type of adverse events in this updated analysis was consistent with that described in the primary analysis; no new safety signals were detected.

Conclusions. OS was not significantly longer in the intent-to-treat population, although there was an improvement in OS in patients with ascites receiving trebananib. PFS- 2 confirmed that the PFS benefit associated with trebananib was maintained through the second disease progression independent of the choice of subsequent therapy.

(c) 2016 Elsevier Inc. All rights reserved.

\section{Introduction}

First-line treatment with a platinum/taxane combination therapy is effective in the treatment of ovarian cancer, but recurrence/relapse is frequent and outcomes are poor, particularly for those patients with a platinum-free interval $<12$ months [1]. In addition to poor overall survival (OS), ovarian cancer is often associated with debilitating symptoms. More than one third of patients diagnosed with the disease have malignant ascites, which can result in abdominal pain, dyspnea, nausea, vomiting, and anorexia [2,3]. Presence of ascites plays a major role in progression of ovarian cancer and is associated with poor prognosis $[2,3]$.

The tumor microenvironment and, specifically, tumor angiogenesis, is involved in ovarian cancer development, progression, and metastasis [4]. Angiogenesis is controlled by growth factors, including the vascular endothelial growth factor (VEGF) pathway and angiopoietin-Tie2 receptor axis. The angiopoietin axis is distinct from the VEGF pathway; angiopoietin 1 (Ang1) and angiopoietin 2 (Ang2) regulate angiogenesis and vascular remodeling by interacting with the endothelial receptor tyrosine kinase, Tie2 [5]. Evidence suggests that the Ang2 pathway plays a role in the pathophysiology of ovarian cancer [6-8] and upregulation of Ang2 is correlated with poor prognosis in women with recurrent ovarian cancer [9]. Several antiangiogenic agents that target the VEGF pathway have been shown to improve progression-free survival (PFS) in patients with ovarian cancer; however, a statistically significant improvement in OS has not been demonstrated [10-18].

Trebananib (AMG 386) is a peptide-Fc fusion protein that binds Ang1 and Ang2, thus preventing their ligand-receptor interaction with Tie2 [19]. In a randomized, placebo-controlled, double-blind, phase 3 study (TRINOVA-1), women with recurrent ovarian cancer receiving paclitaxel plus trebananib $15 \mathrm{mg} / \mathrm{kg}$ once weekly (QW) had significantly prolonged PFS (primary endpoint) compared with patients who received paclitaxel plus placebo ( 7.2 versus 5.4 months; hazard ratio [HR], 0.66; 95\% CI, 0.57-0.77; $P<0.0001$ ) [20] without impairment of quality of life [21]. We report the mature analysis of OS in this study. Additionally, we provide results from a subgroup analysis that evaluated outcomes in patients with/without ascites and an exploratory analysis of time to second disease progression (PFS-2).

\section{Methods}

\subsection{Patients}

Eligibility criteria for TRINOVA-1 have been reported previously [20]. Briefly, women ( $\geq 18$ years) were eligible if they had measurable disease per Response Evaluation Criteria in Solid Tumors (RECIST) version 1.1 [22]; as a result, patients with only ascites or pleural effusion at baseline were excluded from the study. Women were eligible if they had $\leq 3$ prior lines of anticancer therapy, Gynecologic Oncology Group (GOG) performance status $\leq 1$, and platinum-free interval $\leq 12$ months. Women with primary platinum-refractory disease (disease progression during the first 6 cycles or within 6 months after the beginning of primary platinum-based treatment) were excluded. The protocol was approved by each center's independent ethics committee. Patients provided written informed consent.

\subsection{Study design and treatment}

This randomized, double-blind, phase 3 study was conducted at 179 centers globally. Patients were randomly assigned in a 1:1 ratio to receive intravenous paclitaxel $80 \mathrm{mg} / \mathrm{m}^{2}$ once weekly (3 weeks on/1 week off) plus either intravenous trebananib $15 \mathrm{mg} / \mathrm{kg}$ or intravenous placebo once weekly. Randomization was stratified based on platinum-free interval ( $\leq 6$ months versus $>6$ to 12 months), presence/absence of measurable disease per RECIST, and geographic region (North America, Western Europe/Australia, rest of world). Study treatment continued until disease progression, unacceptable toxicity, withdrawal of consent, or death. Dose modifications for paclitaxel were based on attributed toxic effects and allowed for a reduction in dose of $15 \mathrm{mg} / \mathrm{m}^{2}$ per level. Dose reductions for trebananib and placebo were not permitted.

\subsection{Assessments}

Disease was assessed with computed tomography/magnetic resonance imaging of at least the chest, abdomen, and pelvis before cycle 1 and every $8 \pm 1$ weeks for up to 2 years from time of randomization and then every $6 \pm 1$ months thereafter until disease progression. Imaging was evaluated by the investigator per RECIST version 1.1. The presence or absence of ascites at baseline (i.e., within 28 days before randomization) was determined by the investigator at study randomization and recorded using the electronic case report form. Adverse events (AEs) occurring from start of treatment until the safety followup visit (30-37 days after last dose) were recorded and graded using the Common Terminology Criteria for Adverse Events, version 3.0 [23]. Health-related quality of life (HRQoL) was evaluated using Functional Assessment of Cancer Therapy-Ovary (FACT-O) and EQ-5D instruments $[24,25]$.

\subsection{Statistical analyses}

As previously described [20], the planned population size of $900 \mathrm{pa}-$ tients (followed up until at least 510 patients had disease progression or died) was estimated to provide $90 \%$ statistical power to detect a $33 \%$ 
reduction in the hazard of progression or death while limiting the type I error to $5 \%$. A prespecified interim analysis of OS was performed at the time of primary analysis of PFS [20]. Primary analysis of OS was conditional on a demonstration of statistically significant improvement in PFS and was planned for when a minimum of 600 deaths had occurred. With 600 events, the study was estimated to have $85 \%$ statistical power to detect a $28 \%$ improvement in OS while limiting the overall two-sided type I error to 5\%. Differences between arms in PFS and OS were evaluated using a log-rank test, stratified by randomization factors. HRs with 95\% CIs for PFS and OS were calculated using stratified Cox regression models. Baseline characteristics of interest were prespecified in the study's statistical analysis plan. After the study was initiated, the European Medicines Agency (EMA) provided guidance on the use of PFS-2 as a confirmatory measure of the durability of treatment effect, which may be of particular value when multiple lines of therapy are anticipated to limit ability to identify an effect on OS. We evaluated PFS-2 in an exploratory, post hoc analysis, defining it as time from randomization to second disease progression, start of second subsequent therapy, or death from any cause. Analysis of time to second subsequent therapy (time from randomization to administration of second subsequent therapy) was performed to provide a confirmatory evaluation of the PFS-2 outcomes. All efficacy analyses (PFS, OS, PFS-2, time to first/second subsequent therapy) were performed using the intent-to-treat population. Safety analyses included all randomized patients who received $\geq 1$ dose of paclitaxel or trebananib/placebo.

\section{Results}

\subsection{Patient demographics and clinical characteristics}

Between November 10, 2010, and November 19, 2012, 919 women were randomized at 179 sites in 32 countries (Supplemental Fig. 1). The data cutoff date for primary OS analysis was August 29, 2014. Baseline characteristics were balanced across both treatment arms (Table 1). Most patients (61\%) had received $\geq 2$ treatment regimens; $52 \%$ of all patients had a platinum-free interval of $\geq 0$ to $\leq 6$ months. Median (range) follow-up time for trebananib and placebo treatment arms was 18.0 (0-43) months and 17.5 (0-44) months, respectively.

Median (range) number of cycles administered was 6.0 (1-37) in the trebananib arm and $5.0(1-43)$ in the placebo arm. Median relative dose intensity for paclitaxel was $93 \%$ and $92 \%$ in the trebananib and placebo treatment arms, respectively. The median relative dose intensity for trebananib was $97 \%$. Patients in both arms received extensive anticancer therapy after ceasing study treatment. Overall, 331 patients (72\%) in the trebananib arm and 353 patients (77\%) in the placebo arm received $\geq 1$ subsequent anticancer therapy after disease progression; 201 patients (44\%) in the trebananib arm and 231 (50\%) in the placebo arm received $\geq 2$ subsequent anticancer therapies. Treatments administered included platinum agents (trebananib arm, $\mathrm{n}=198$ [60\%]; placebo arm, $\mathrm{n}=205$ [58\%]), other cytotoxic agents (trebananib arm, $\mathrm{n}=313$ [95\%]; placebo arm, $\mathrm{n}=331$ [94\%]), and other antiangiogenic therapy (trebananib arm, $n=51$ [15\%]; placebo arm, $\mathrm{n}=48$ [14\%]; Supplemental Table 1). Median (range) number of subsequent anticancer therapies administered was 2 (1-8) in both arms.

\subsection{Overall survival and mature progression-free survival}

In the primary analysis of the intent-to-treat population, paclitaxel plus trebananib did not significantly improve OS compared with paclitaxel plus placebo. At the time of analysis, 315 patients (68\%) in the trebananib arm and 313 patients (68\%) in the placebo arm had died. Median OS was 19.3 (95\% CI, 17.6-21.4) months for paclitaxel plus trebananib and 18.3 (95\% CI, 16.3-20.4) months for paclitaxel plus placebo (HR, $0.95 ; 95 \% \mathrm{CI}, 0.81-1.11 ; P=0.52$; Fig. $1 \mathrm{~A}$ ). Evaluation of OS outcomes in subgroups showed the most notable effect among patients with ascites at baseline (Fig. 1B). An updated analysis of PFS
Table 1

Baseline demographics and clinical characteristics.

\begin{tabular}{|c|c|c|}
\hline & $\begin{array}{l}\text { Paclitaxel + Placebo } \\
n=458\end{array}$ & $\begin{array}{l}\text { Paclitaxel + Trebananib } \\
15 \mathrm{mg} / \mathrm{kg} \text { QW } \\
\mathrm{n}=461\end{array}$ \\
\hline Median (range) age, years & $59(22-84)$ & $60(29-84)$ \\
\hline \multicolumn{3}{|l|}{ Race/ethnicity, n (\%) } \\
\hline White & $363(79)$ & $387(84)$ \\
\hline Asian & $82(18)$ & $58(13)$ \\
\hline Other, including black ethnicity & $13(3)$ & $16(3)$ \\
\hline \multicolumn{3}{|l|}{ Region, $\mathrm{n}(\%)$} \\
\hline North America & $91(20)$ & $93(20)$ \\
\hline Western Europe/Australia & $189(41)$ & $193(42)$ \\
\hline Rest of world & $178(39)$ & $175(38)$ \\
\hline \multicolumn{3}{|l|}{ GOG performance status, n (\%) } \\
\hline 0 & $252(55)$ & $259(56)$ \\
\hline 1 & $205(45)$ & $200(43)$ \\
\hline $2^{\mathrm{a}}$ & $1(<1)$ & $2(<1)$ \\
\hline \multicolumn{3}{|l|}{ Primary tumor type, n (\%) } \\
\hline Ovarian cancer & $419(91)$ & $423(92)$ \\
\hline Primary peritoneal carcinoma & $24(5)$ & $24(5)$ \\
\hline Fallopian tube cancer & $15(3)$ & $14(3)$ \\
\hline \multicolumn{3}{|l|}{ Histologic type, n (\%) } \\
\hline Serous & $388(85)$ & $385(84)$ \\
\hline Endometrioid & $26(6)$ & $29(6)$ \\
\hline Other ${ }^{\mathrm{b}}$ & $44(10)$ & $47(10)$ \\
\hline \multicolumn{3}{|l|}{ Histologic grade, n (\%) } \\
\hline Well differentiated & $31(7)$ & $24(5)$ \\
\hline Moderately differentiated & $84(18)$ & $69(15)$ \\
\hline Poorly differentiated & $256(56)$ & $274(59)$ \\
\hline Unknown & $87(19)$ & $94(20)$ \\
\hline \multicolumn{3}{|l|}{ Prior lines of therapy, $\mathrm{n}(\%)$} \\
\hline 1 & $172(38)$ & $190(41)$ \\
\hline 2 & $172(38)$ & $174(38)$ \\
\hline 3 & $114(25)$ & $95(21)$ \\
\hline $4^{\mathrm{a}}$ & $0(0)$ & $2(<1)$ \\
\hline \multicolumn{3}{|l|}{ Platinum-free interval, $\mathrm{n}(\%)^{\mathrm{c}}$} \\
\hline$\leq 6$ months & $245(53)$ & $235(51)$ \\
\hline$>6$ to $\leq 12$ months & $212(46)$ & $223(48)$ \\
\hline $\begin{array}{l}\text { Measurable disease at baseline, } \\
\mathrm{n}(\%)\end{array}$ & 433 (95) & 436 (95) \\
\hline Baseline ascites, $\mathrm{n}(\%)$ & $141(31)$ & $154(34)$ \\
\hline
\end{tabular}

GOG, Gynecologic Oncology Group; QW, once weekly.

a Protocol deviation.

b Includes undifferentiated and transitional. Clear cell and mucinous histologies were excluded by protocol.

c Four patients were refractory (protocol deviation).

performed at the time of the primary analysis of OS confirmed the previously reported [20] significant prolongation of PFS in the trebananib arm: median PFS in the trebananib arm was 7.4 (95\% CI, 7.0-7.8) months versus $5.4(95 \% \mathrm{CI}, 4.7-5.5)$ months in the placebo arm (HR, $0.70 ; 95 \% \mathrm{CI}, 0.61-0.80 ; P<0.001)$. In a sensitivity analysis, median time to first subsequent treatment or death (a proxy for PFS) was 7.5 months for the trebananib arm versus 6.0 months for the placebo arm (HR, 0.82; 95\% CI, 0.71-0.94; $P=0.004)$. For PFS, HRs favored the trebananib arm for all baseline characteristics evaluated (Fig. 1C).

\subsection{Exploratory subgroup analysis of patients with ascites at baseline}

At baseline, 295 patients had ascites and 624 patients did not have ascites. Demographic/clinical characteristics were similar for both patient groups (Table 2). At the time of this analysis, 107/141 patients (76\%) with ascites in the trebananib treatment arm and 134/154 patients (87\%) with ascites in the placebo arm had died. In this subgroup analysis, paclitaxel plus trebananib significantly prolonged OS in patients with ascites compared with paclitaxel plus placebo treatment. Among patients with ascites, median OS in the trebananib arm was 14.5 (95\% CI, 11.4-18.6) months versus 12.3 (95\% CI, 9.1-13.9) months in the placebo arm (HR, $0.72 ; 95 \% \mathrm{Cl}, 0.55-0.93 ; P=0.011 ;$ Fig. 2 ). There was little evidence that trebananib treatment was associated with a 
A

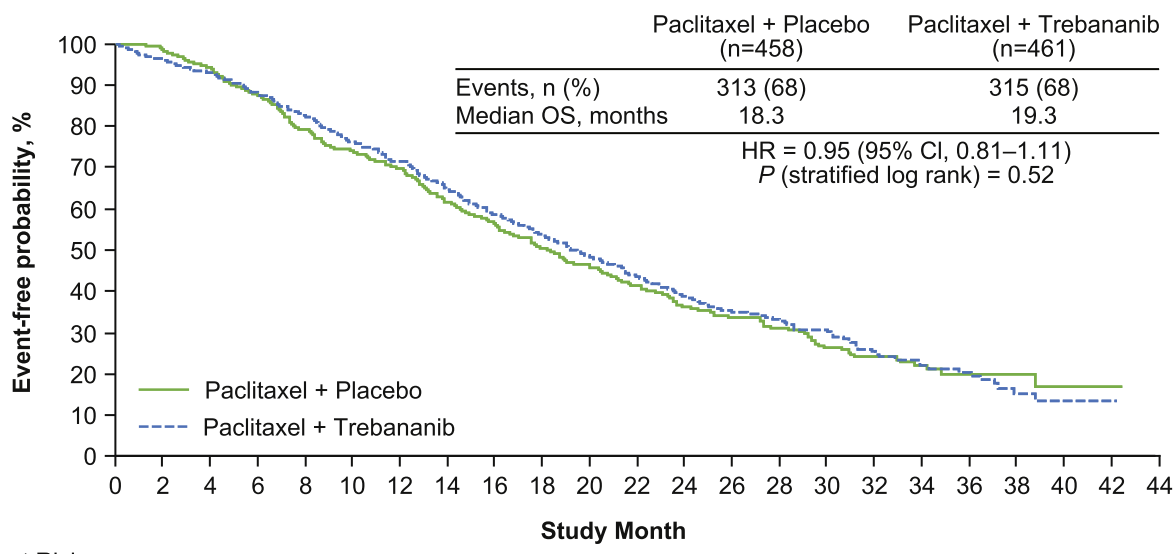

Patients at Risk

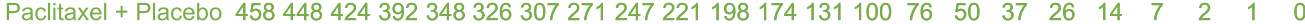
Paclitaxel + Trebananib $4614404233953663363142842562342101811411108263 \quad 44 \quad 3123 \quad 12 \quad 6 \quad 1 \quad 0$

B

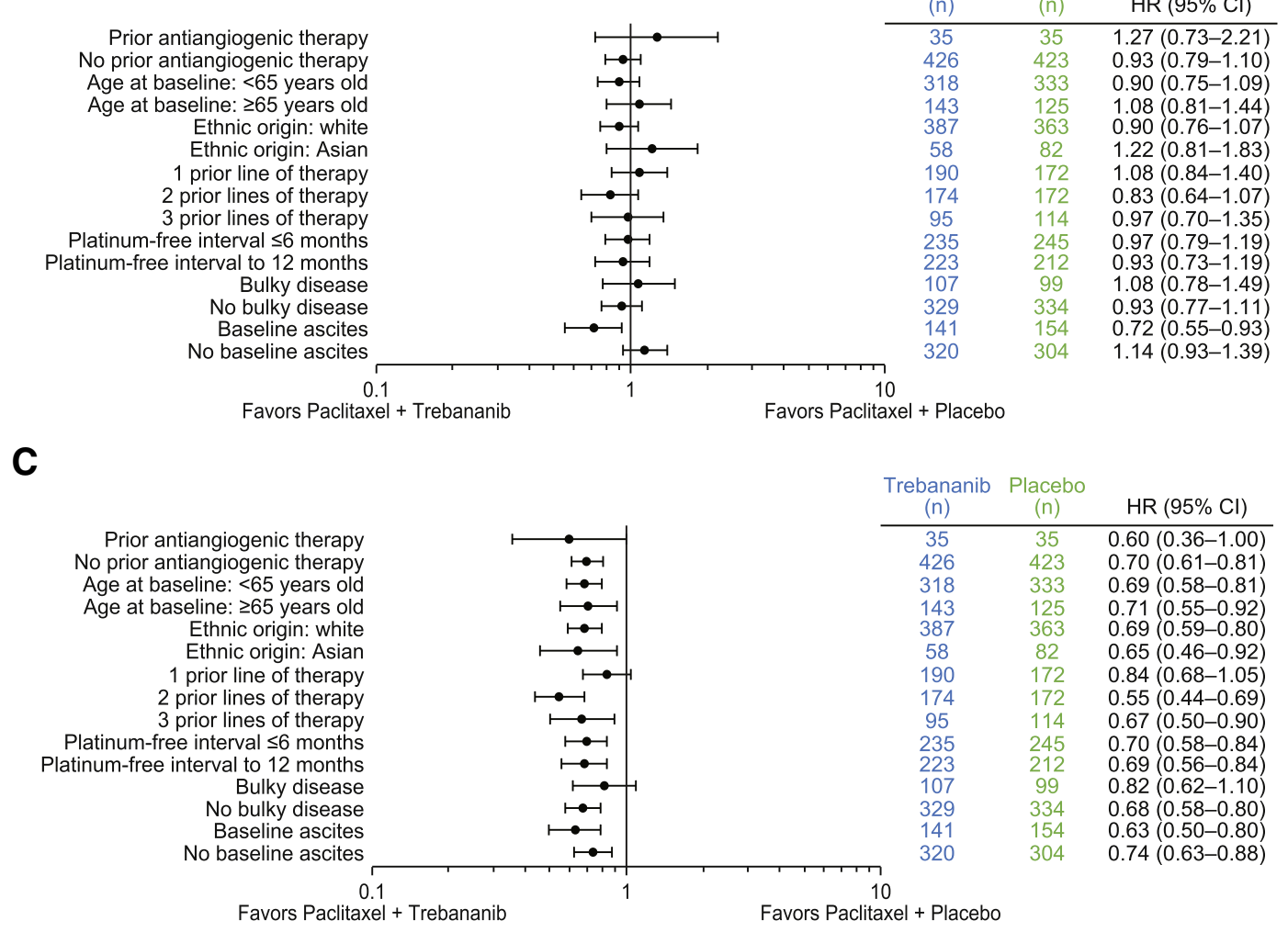

Fig. 1. Overall survival in intent-to-treat population (A), overall survival (B), and progression-free survival (C) in subgroups of baseline characteristics. HR, hazard ratio.

decrement in $\mathrm{HRQ} o \mathrm{~L}$ in patients with ascites. Indeed, there appeared to be a trend toward improvement in some instruments (Supplemental Fig. 2). Among patients without ascites, there was no significant difference in OS between treatment arms. Median OS in the trebananib arm was 21.4 (95\% CI, 19.1-23.6) months compared with 22.8 (95\% CI, 20.5-25.9) months in the placebo arm (HR, 1.14; 95\% CI, 0.93-1.39; $P=0.20$ ) (Supplemental Fig. 3).

\subsection{Time to second disease progression}

To evaluate the durability of the effect of trebananib treatment on PFS, we performed an exploratory analysis of PFS-2 (i.e., time to second disease progression; Fig. 3A). At the time of analysis, 405/461 patients (88\%) in the trebananib arm and $413 / 458$ patients (90\%) in the placebo arm had had a PFS-2 event (i.e., a second disease progression after randomization into the study). Median PFS-2 was 12.5 (95\% CI, 7.3-18.5) and 10.9 (95\% CI, 10.0-11.5) months for the trebananib and placebo arms, respectively (HR, 0.85; 95\% CI, 0.74-0.98; $P=0.024$; Fig. 3B). In a sensitivity analysis, time to second subsequent treatment was 13.4 months for the trebananib arm versus 11.7 months for the placebo $\operatorname{arm}(\mathrm{HR}, 0.83 ; 95 \% \mathrm{Cl}, 0.72-0.96 ; P=0.011)$.

\subsection{Adverse events}

Among patients who received $\geq 1$ dose of study medication, $97 \%$ in the trebananib arm and $96 \%$ in the placebo arm had treatmentemergent AEs of any grade (Table 3 ). The proportion of patients with grade $\geq 3 \mathrm{AEs}$, serious AEs, or fatal AEs was similar in both treatment 
Table 2

Demographics of patients with and without ascites.

\begin{tabular}{|c|c|c|c|c|c|c|}
\hline & \multicolumn{3}{|c|}{ Patients with ascites at baseline } & \multicolumn{3}{|c|}{ Patients without ascites at baseline } \\
\hline & $\begin{array}{l}\text { Paclitaxel } \\
+ \text { Placebo } \\
\mathrm{n}=154\end{array}$ & $\begin{array}{l}\text { Paclitaxel + Trebananib } \\
15 \mathrm{mg} / \mathrm{kg} \text { QW } \\
\mathrm{n}=141\end{array}$ & $\begin{array}{l}\text { All Patients } \\
\mathrm{n}=295\end{array}$ & $\begin{array}{l}\text { Paclitaxel } \\
+ \text { Placebo } \\
\mathrm{n}=304\end{array}$ & $\begin{array}{l}\text { Paclitaxel + Trebananib } \\
15 \mathrm{mg} / \mathrm{kg} \mathrm{QW} \\
\mathrm{n}=320\end{array}$ & $\begin{array}{l}\text { All Patients } \\
\mathrm{n}=624\end{array}$ \\
\hline Median (range) age, years & $58(32-80)$ & $59(36-80)$ & $58.0(32-80)$ & $59(22-84)$ & $60(29-84)$ & $60.0(22-84)$ \\
\hline \multicolumn{7}{|l|}{ GOG performance status, n (\%) } \\
\hline 0 & $73(47)$ & $61(43)$ & $134(45)$ & $179(59)$ & $198(62)$ & $377(60)$ \\
\hline 1 & $80(52)$ & $79(56)$ & $159(54)$ & $125(41)$ & $121(38)$ & $246(39)$ \\
\hline $2^{\mathrm{a}}$ & $1(<1)$ & $1(<1)$ & $2(1)$ & 0 & $1(<1)$ & $1(<1)$ \\
\hline \multicolumn{7}{|l|}{ Primary tumor type, n (\%) } \\
\hline Ovarian cancer & $140(91)$ & $125(89)$ & $265(90)$ & $279(92)$ & $298(93)$ & $577(93)$ \\
\hline Primary peritoneal carcinoma & $9(6)$ & $10(7)$ & $19(6)$ & $15(5)$ & $14(4)$ & $29(5)$ \\
\hline Fallopian tube cancer & $5(3)$ & $6(4)$ & $11(4)$ & $10(3)$ & $8(3)$ & $18(3)$ \\
\hline \multicolumn{7}{|l|}{ Histologic grade, n (\%) } \\
\hline Well differentiated & $10(6)$ & $6(4)$ & $16(5)$ & $21(7)$ & $18(6)$ & $39(6)$ \\
\hline Moderately differentiated & $24(16)$ & $22(16)$ & $46(16)$ & $60(20)$ & $47(15)$ & $107(17)$ \\
\hline Poorly differentiated & $86(56)$ & $83(59)$ & $169(57)$ & $170(56)$ & $191(60)$ & $361(58)$ \\
\hline Unknown & $34(22)$ & $30(21)$ & $64(22)$ & $53(17)$ & $64(20)$ & $117(19)$ \\
\hline \multicolumn{7}{|l|}{ Prior lines of therapy, $\mathrm{n}(\%)$} \\
\hline 1 & $62(40)$ & $60(43)$ & $122(41)$ & $110(36)$ & $130(41)$ & 240 (39) \\
\hline 2 & $61(40)$ & $47(33)$ & $108(37)$ & $111(37)$ & $127(40)$ & $238(38)$ \\
\hline 3 & $31(20)$ & $33(23)$ & $64(22)$ & $83(27)$ & $62(19)$ & $145(23)$ \\
\hline $4^{a}$ & 0 & $1(<1)$ & $1(<1)$ & 0 & $1(<1)$ & $1(<1)$ \\
\hline \multicolumn{7}{|l|}{ Prior antiangiogenic therapy, n (\%) } \\
\hline Yes & $10(6)$ & $11(8)$ & $21(7)$ & $25(8)$ & $24(8)$ & $49(8)$ \\
\hline No & $144(94)$ & $130(92)$ & $274(93)$ & $279(92)$ & $296(93)$ & $575(92)$ \\
\hline \multicolumn{7}{|l|}{ Platinum-free interval, $\mathrm{n}(\%)^{\mathrm{b}}$} \\
\hline$\leq 6$ months & $94(61)$ & $78(55)$ & $172(58)$ & $151(50)$ & $157(49)$ & $308(49)$ \\
\hline$>6$ to $\leq 12$ months & $60(39)$ & $63(45)$ & $123(42)$ & $152(50)$ & $160(50)$ & $312(50)$ \\
\hline Measurable disease at baseline, $\mathrm{n}(\%)$ & $143(93)$ & $131(93)$ & $274(93)$ & $290(95)$ & $305(95)$ & $595(95)$ \\
\hline
\end{tabular}

GOG, Gynecologic Oncology Group; QW = once weekly.

a Protocol violation.

b Four patients were refractory (protocol deviation).

arms. However, treatment with paclitaxel plus trebananib was associated with more AE-related treatment discontinuations (22\%) than treatment with paclitaxel plus placebo (8\%), although this finding must be considered in the context of the higher risk of disease progression in the paclitaxel plus placebo arm.

The proportion of patients who developed edema of any grade during treatment was greater in the trebananib arm (66\%) than in the placebo arm (30\%). The most frequently occurring edema events were localized edema (59\% versus $27 \%$ ) and generalized edema (12\% versus $3 \%$; Table 3). More patients in the trebananib arm $(n=52 ; 11 \%)$ than in the placebo $\operatorname{arm}(\mathrm{n}=4 ; 1 \%)$ had grade $\geq 3$ edema. There was one grade 5 event of generalized edema in the setting of diabetic ketoacidosis and neutropenic sepsis in the trebananib arm; no other grade 4/5 edema events occurred during the study. Several other AEs presented with a higher incidence rate in the trebananib arm. Ascites of any grade ( $22 \%$ versus $13 \%$ ) and grade 3 (13\% versus $8 \%$ ) occurred more frequently with trebananib treatment than with placebo. There were no grade $4 / 5$ ascites events. Pleural effusion, nasopharyngitis, and weight increase also occurred more frequently with trebananib treatment (Table 3). AEs of interest were hypertension (trebananib, $7 \%$ versus placebo, $4 \%$ ), pulmonary embolism ( $2.2 \%$ versus $1.1 \%$ ), arterial thrombotic events $(0.7 \%$ in both arms), venous thromboembolic events

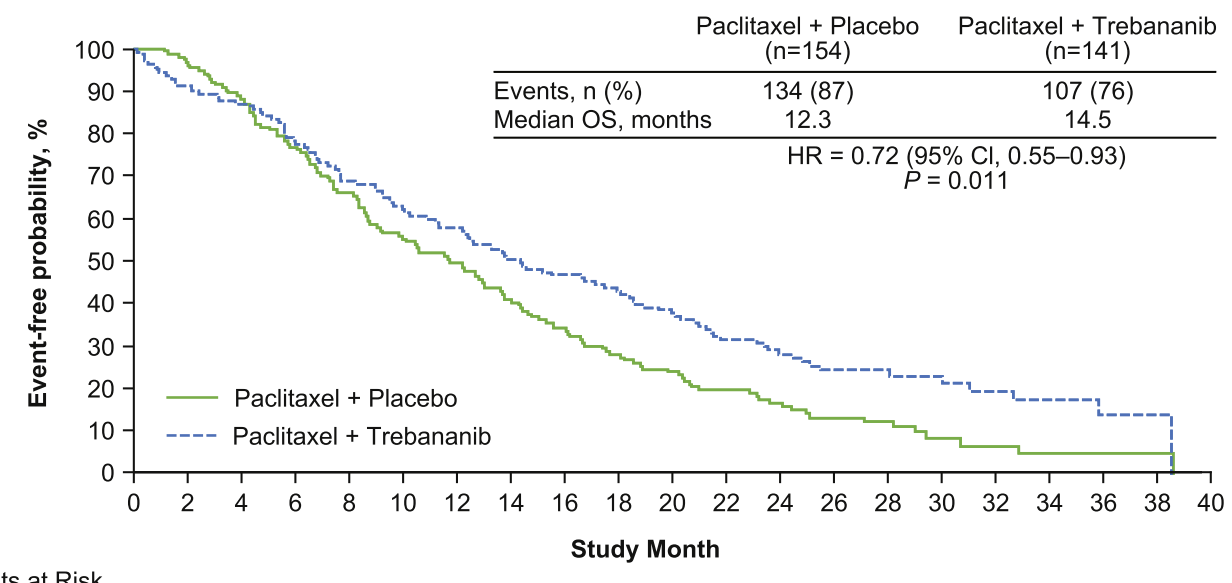

Patients at Risk

Paclitaxel + Placebo $154 \begin{array}{lllllllllllllllllllllll}148 & 135 & 115 & 98 & 83 & 74 & 61 & 51 & 42 & 36 & 28 & 21 & 14 & 11 & 6 & 4 & 3 & 2 & 2 & 0\end{array}$

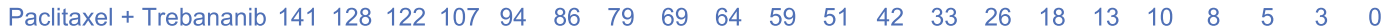

Fig. 2. Overall survival in patients with ascites at baseline. HR, hazard ratio; OS, overall survival. 
A

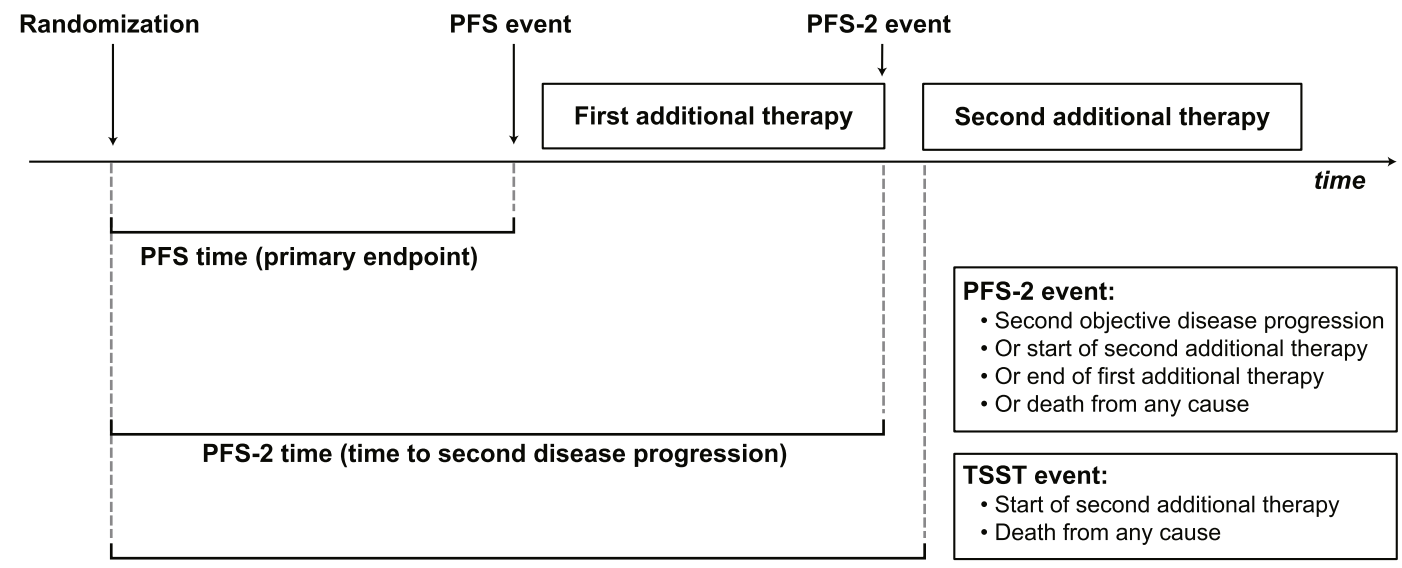

TSST (time to second subsequent therapy)

B

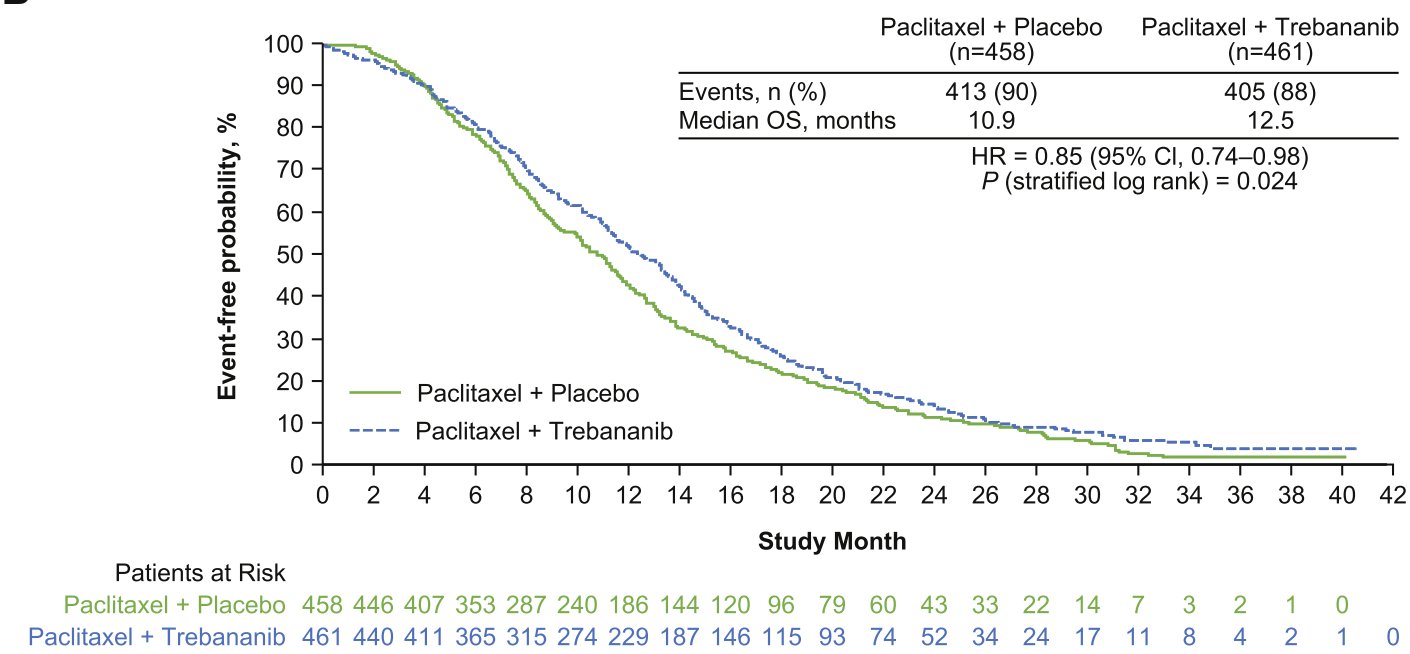

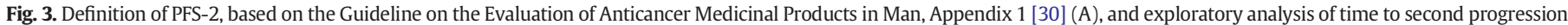
event (B). HR, hazard ratio; OS, overall survival; PFS, progression-free survival; PFS-2, time to second progression event; TSST, time to second subsequent treatment.

(7.4\% versus $4.4 \%$ ), gastrointestinal perforation (2.0\% versus $0.2 \%$ ), impaired wound healing (2.2\% versus $1.5 \%)$, and blurred vision $(6.9 \%$ versus $4.4 \%$ ).

Table 3

Adverse events.

\begin{tabular}{lll}
\hline Patients with adverse events, $\mathrm{n}(\%)$ & $\begin{array}{l}\text { Paclitaxel } \\
+ \text { Placebo } \\
\mathrm{n}=452\end{array}$ & $\begin{array}{l}\text { Paclitaxel } \\
+ \text { Trebananib } \\
15 \mathrm{mg} / \mathrm{kg} \text { QW } \\
\mathrm{n}=461\end{array}$ \\
\hline $\begin{array}{ll}\text { Any treatment-emergent adverse event } \\
\text { Grade } \geq 3\end{array}$ & $434(96)$ & $449(97)$ \\
Grade $\geq 4$ & $199(44)$ & $223(48)$ \\
Grade 5 & $36(8)$ & $29(6)$ \\
Serious adverse event & $20(4)$ & $26(6)$ \\
Adverse event leading to discontinuation & $138(31)$ & $167(36)$ \\
of trebananib & $34(8)$ & $101(22)$ \\
Treatment-emergent adverse events with $\geq 5 \%$ & & \\
difference in incidence between arms & & \\
Localized edema & $122(27)$ & $272(59)$ \\
Pleural effusion & $18(4)$ & $67(15)$ \\
Ascites & $57(13)$ & $101(22)$ \\
Generalized edema & $12(3)$ & $54(12)$ \\
Weight increased & $10(2)$ & $44(10)$ \\
Nasopharyngitis & $31(7)$ & $59(13)$ \\
Neutropenia & $129(29)$ & $104(23)$ \\
Anemia & $94(21)$ & $50(11)$ \\
\hline
\end{tabular}

QW, once weekly.

\section{Discussion}

In the TRINOVA-1 study, the combination of paclitaxel plus trebananib $15 \mathrm{mg} / \mathrm{kg}$ QW significantly prolonged PFS (the study's primary endpoint) compared with paclitaxel plus placebo in women with recurrent ovarian cancer, with benefit across most patient subgroups evaluated [20]. Although treatment with paclitaxel plus trebananib did not significantly prolong median OS in the intent-to-treat population (HR, 0.95; 18.3 versus 19.3 months; $P=0.52$ ), there was a significant improvement in median OS favoring the trebananib arm among patients with ascites at baseline (HR, $0.72 ; 14.5$ versus 12.3 months; $P=0.011$ ). There was a significant improvement in median PFS in this analysis that was consistent with that of the primary analysis of PFS [20]. Similarly, the incidence and nature of AEs was consistent with that described in the primary analysis of PFS; there were no new safety signals [20].

Overall survival has historically been regarded as among the most objective and clinically relevant endpoints in cancer studies. However, it has proven exceptionally difficult to show a statistically significant prolongation of OS with the addition of targeted therapy to standard treatments in patients with ovarian cancer [10-18,26-28]. Potential confounding factors for OS include the long postprogression survival period, the administration of multiple postprogression therapies, and the potential for postprogression crossover [27,29]. In the TRINOVA-1 study, $47 \%$ of patients had received at least two lines of postprogression therapy, and $14 \%$ received an antiangiogenic therapy after progression. 
One approach to addressing these challenges suggested by the EMA is to assess the durability of treatment effect by evaluating postprogression endpoints such as PFS-2 and time to second subsequent therapy (TSST) $[27,30]$. The TRINOVA- 1 study is among the first to demonstrate that an antiangiogenic therapy significantly delayed the time to second progression (i.e. PFS-2) in women with recurrent ovarian cancer. Consistent with the exploratory analysis of PFS-2, a confirmatory analysis that evaluated TSST also showed benefit in the trebananib arm. These results indicate that the PFS benefit achieved with trebananib was maintained through at least the second disease progression following enrollment in the study irrespective of the second therapy administered. Patients received multiple lines of postprogression therapy (up to 8 lines, including both chemotherapy and targeted agents), which are likely to have confounded the analysis of OS. The data suggest that, although a significant improvement in OS was not achieved in the intent-to-treat population, trebananib provided a clinically meaningful benefit and that this benefit was maintained at least through the second disease progression, without impairment of quality of life [21].

A second approach to the challenges of demonstrating an OS benefit in recurrent ovarian cancer is to evaluate outcomes in patient subgroups likely to derive particular benefit from treatment. In a subgroup analysis, we found that median OS was significantly longer in patients with ascites at baseline who received paclitaxel plus trebananib compared with patients who received paclitaxel plus placebo. Although PFS was improved among patients without ascites at baseline, this benefit was not maintained postprogression. As has been observed in other studies in patients with ovarian cancer [31,32], there was no significant difference in OS between treatment arms in patients without ascites. The presence of malignant ascites in advanced ovarian cancer has been associated with disease recurrence and poor prognosis [33,34], and we found that median OS was much shorter in patients with ascites (trebananib, 14.5 months; placebo, 12.3 months) than those without (trebananib, 22.8 months; placebo, 21.4 months) irrespective of treatment arm $[33,34]$.

Evidence indicates that angiogenic pathways play an important role in the formation of ascites in ovarian cancer $[9,33]$. The presence of ascites at baseline has been shown to be associated with poor prognosis and is considered a sign of peritoneal carcinomatosis [3]. Peritoneal carcinomatosis has been shown to be particularly dependent on angiogenesis and as a result, may be susceptible to antiangiogenic therapy [33]. In this context, it is notable that improvements in outcomes have also been observed in studies with other antiangiogenic agents in ovarian cancer patients with ascites. Specifically, subgroup analyses showed improved OS in patients with ovarian cancer who received bevacizumab in the AURELIA (recurrent disease) [17], GOG 218 (firstline) [32], and ICON7 (first-line) [18] phase 3 clinical studies. Among patients with ascites at baseline in the AURELIA study $(n=113)$, there was a trend toward longer median OS among patients who received bevacizumab plus chemotherapy compared with patients who received placebo plus chemotherapy in the subgroup of patients with ascites at baseline (11.7 versus 7.9 months; HR, 0.67; 95\% CI, 0.45-1.00) [31]. Although angiogenic factors may play a role in ascites formation $[35,36]$, there are other factors that may also lead to formation of ascites in ovarian cancer. Although a subgroup analysis, it must be noted that TRINOVA-1 was of sufficient size overall that the ascites subgroup analysis contained 295 patients in which an OS benefit was shown. Results from this study suggest that use of ascites as a stratification factor in future trials of antiangiogenic agents in ovarian cancer may be appropriate.

The AEs reported at the time of the mature OS analysis were consistent with those previously reported in the primary analysis [20], as would be anticipated given the limited additional exposure. The most common $\mathrm{AE}$ associated with trebananib treatment was low-grade edema, which was also described in both the TRINOVA-1 primary analysis [20] and in other trebananib studies [37]. Incidences of AEs common to anti-VEGF therapies, such as hypertension, hemorrhage, proteinuria, thrombotic events, and pulmonary embolisms, were not significantly increased in the trebananib treatment group compared with placebo, which is consistent with the toxicity profile of trebananib reported in previous studies [37].

In summary, paclitaxel plus weekly trebananib did not significantly prolong OS in the intent-to-treat population compared with paclitaxel plus placebo. As in the previously reported primary analysis, PFS remained significantly longer with trebananib treatment. In an exploratory subgroup analysis, we showed that both PFS and OS were improved with trebananib in the clinically important subgroup of patients with ascites at baseline. This suggests that trebananib, as other antiangiogenic drugs, may have particular value in the group of poor prognosis patients who have ascites. Additionally, consistent with guidance from the EMA, we evaluated PFS- 2 as an exploratory endpoint and demonstrated that the PFS benefit associated with trebananib was durable and was maintained through at least the second disease progression independent of the choice of subsequent therapy.

\section{Conflicts of interest}

BJ Monk: grants and/or personal fees paid to his institution from Amgen, Novartis, Eli Lilly Genentech, Janssen/Johnson \& Johnson, Array, TESARO and Morphotek; honoraria for speaker bureaus from AstraZeneca, Myriad, Janssen/Johnson \& Johnson and Roche/Genentech; consultant for Roche/Genentech, Merck, TESARO, AstraZeneca, Gradalis, Cerulean, Amgen, Vermillion, ImmunoGen, Pfizer, Bayer, NuCana, Insys, GlaxoSmithKline, Verastem, and Clovis. A Poveda: consulting and advisory roles for Roche and AstraZeneca; travel and accommodation expenses from PharmaMar. I Vergote: consulting and advisory role from Amgen. F Raspagliesi: grants/personal fees for participation in advisory boards for Amgen, Roche and PharmaMar. K Fujiwara: grants from Amgen; grants/personal fees from Pfizer, Glaxo SmithKline, Taiho, Zeria, Chugai, Eisai, and MSD; grants from Sanofi and Eli Lilly; and personal fees from Daiichi Sankyo and Yakult. C Lhommé: grants/personal fees paid to her institution by Amgen and Roche. RL Coleman: travel expenses for participation on the TRINOVA- 1 study steering committee. A Bamias: reported fees from Amgen Inc., during the conduction and participation in the study and personal fees outside of the submitted work. M Fabbro: research funding paid to his institution by Amgen. H Ma, FD Vogl, and BA Bach: employees of and stock holders in Amgen Inc. AM Oza: research funding from Amgen Inc. DS Bae, A Oaknin, I Ray-Coquard, DM Provencher, BY Karlan, G Richardson, C Marth, D Gallardo Rincón, A Brize, A Redondo: no conflicts to disclose.

\section{Acknowledgments}

The authors thank Jennifer Venzie, PhD, and Ali Hassan, PhD (Complete Healthcare Communications, LLC, Chadds Ford, PA), for assistance in the preparation of this manuscript, and Kathy Zhang (Amgen Inc.) for statistical support.

\section{Appendix A. Supplementary data}

Supplementary data to this article can be found online at http://dx. doi.org/10.1016/j.ygyno.2016.07.112.

\section{References}

[1] N. Colombo, Optimizing treatment of the partially platinum-sensitive ovarian cancer patient, Future Oncol. 9 (2013) 19-23.

[2] S. Barni, M. Cabiddu, M. Ghilardi, F. Petrelli, A novel perspective for an orphan problem: old and new drugs for the medical management of malignant ascites, Crit. Rev. Oncol. Hematol. 79 (2011) 144-153.

[3] S.L. Sangisetty, T.J. Miner, Malignant ascites: a review of prognostic factors, pathophysiology and therapeutic measures, World J. Gastrointest. Surg. 4 (2012) 87-95.

[4] R.L. Coleman, B.J. Monk, A.K. Sood, T.J. Herzog. Latest research and treatment of advanced-stage epithelial ovarian cancer, Nat. Rev. Clin. Oncol. 10 (2013) 211-224.

[5] N.G. Gavalas, M. Liontos, S.P. Trachana, et al., Angiogenesis-related pathways in the pathogenesis of ovarian cancer, Int. J. Mol. Sci. 14 (2013) 15885-15909. 
[6] A.M. Martoglio, B.D. Tom, M. Starkey, A.N. Corps, D.S. Charnock-Jones, S.K. Smith, Changes in tumorigenesis- and angiogenesis-related gene transcript abundance profiles in ovarian cancer detected by tailored high density cDNA arrays, Mol. Med. 6 (2000) 750-765.

[7] L. Zhang, N. Yang, J.W. Park, et al., Tumor-derived vascular endothelial growth factor up-regulates angiopoietin-2 in host endothelium and destabilizes host vasculature, supporting angiogenesis in ovarian cancer, Cancer Res. 63 (2003) 3403-3412.

[8] K. Hata, J. Udagawa, R. Fujiwaki, K. Nakayama, H. Otani, K. Miyazaki, Expression of angiopoietin-1, angiopoietin-2, and Tie2 genes in normal ovary with corpus luteum and in ovarian cancer, Oncology 62 (2002) 340-348.

[9] H. Sallinen, T. Heikura, J. Koponen, et al., Serum angiopoietin-2 and soluble VEGFR-2 levels predict malignancy of ovarian neoplasm and poor prognosis in epithelial ovarian cancer, BMC Cancer 14 (2014) 696.

[10] E. Pujade-Lauraine, F. Hilpert, B. Weber, et al., Bevacizumab combined with chemotherapy for platinum-resistant recurrent ovarian cancer: the AURELIA open-label randomized phase III trial, J. Clin. Oncol. 32 (2014) 1302-1308.

[11] C. Aghajanian, S.V. Blank, B.A. Goff, et al., OCEANS: a randomized, double-blind, placebo-controlled phase III trial of chemotherapy with or without bevacizumab in patients with platinum-sensitive recurrent epithelial ovarian, primary peritoneal, or fallopian tube cancer, J. Clin. Oncol. 30 (2012) 2039-2045.

[12] R.L. Coleman, M.F. Brady, T.J. Herzog, et al., A phase III randomized controlled clinical trial of carboplatin and paclitaxel alone or in combination with bevacizumab followed by bevacizumab and secondary cytoreductive surgery in platinum-sensitive, recurrent ovarian, peritoneal primary and fallopian tube cancer (Gynecologic Oncology Group 0213) [abstract], Gynecol. Oncol. 137 (2015) 3-4.

[13] R.A. Burger, M.F. Brady, M.A. Bookman, et al., Incorporation of bevacizumab in the primary treatment of ovarian cancer, N. Engl. J. Med. 365 (2011) 2473-2483.

[14] T.J. Perren, A.M. Swart, J. Pfisterer, et al., A phase 3 trial of bevacizumab in ovarian cancer, N. Engl. J. Med. 365 (2011) 2484-2496.

[15] A. Du Bois, G. Kristensen, I. Ray-Coquard, et al., AGO-OVAR 12: a randomized placebo-controlled GCIG/ENGOT-intergroup phase III trial of standard frontline chemotherapy \pm nintedanib for advanced ovarian cancer [abstract], Int. J. Gynecol. Cancer 23 (2013) PL01.

[16] A. du Bois, A. Floquet, J.W. Kim, et al., Incorporation of pazopanib in maintenance therapy of ovarian cancer, J. Clin. Oncol. 32 (2014) 3374-3382.

[17] A.M. Poveda, F. Selle, F. Hilpert, et al., Bevacizumab combined with weekly paclitaxel, pegylated liposomal doxorubicin, or topotecan in platinum-resistant recurrent ovarian cancer: analysis by chemotherapy cohort of the randomized phase III AURELIA trial, J. Clin. Oncol. 33 (2015) 3836-3838.

[18] A.M. Oza, A.D. Cook, J. Pfisterer, et al., Standard chemotherapy with or without bevacizumab for women with newly diagnosed ovarian cancer (ICON7): overall survival results of a phase 3 randomised trial, Lancet Oncol. 16 (2015) 928-936.

[19] J. Oliner, H. Min, J. Leal, et al., Suppression of angiogenesis and tumor growth by selective inhibition of angiopoietin-2, Cancer Cell 6 (2004) 507-516.

[20] B.J. Monk, A. Poveda, I. Vergote, et al., Anti-angiopoietin therapy with trebananib for recurrent ovarian cancer (TRINOVA-1): a randomised, multicentre, double-blind, placebo-controlled phase 3 trial, Lancet Oncol. 15 (2014) 799-808.

[21] F.L.C. Raspagliesi, B.J. Monk, R.L. Coleman, T.J. Herzog, L. Navale, D.J. Warner, K. Fujiwara, Health-related quality-of-life analysis from the TRINOVA-1 study of weekly paclitaxel plus trebananib or placebo in women with recurrent ovarian cancerPresented at: 45th Annual Meeting on Women's Cancer; Mar 22-25, 2014 2014 (Tampa, FL).

[22] E.A. Eisenhauer, P. Therasse, J. Bogaerts, et al., New response evaluation criteria in solid tumours: revised RECIST guideline (version 1.1), Eur. J. Cancer 45 (2009) 228-247.

[23] Cancer Therapy Evaluation Program, Common Terminology Criteria for Adverse Events v3.0 (CTCAE), http://ctep.cancer.gov/protocoldevelopment/electronic_applications/docs/ctcaev3.pdf.

[24] R. Brooks, EuroQol: the current state of play, Health Policy 37 (1996) 53-72.

[25] D.F. Cella, D.S. Tulsky, G. Gray, et al., The functional assessment of cancer therapy scale: development and validation of the general measure, J. Clin. Oncol. 11 (1993) 570-579.

[26] US Department of Health and Human Services, Guidance for Industry: Clinical Trial Endpoints for the Approval of Cancer Drugs and Biologics(Rockville, MD May 2007) 2007.

[27] U.A. Matulonis, A.M. Oza, T.W. Ho, J.A. Ledermann, Intermediate clinical endpoints: a bridge between progression-free survival and overall survival in ovarian cancer trials, Cancer 121 (2015) 1737-1746.

[28] K.R. Broglio, D.A. Berry, Detecting an overall survival benefit that is derived from progression-free survival, J. Natl. Cancer Inst. 101 (2009) 1642-1649.

[29] T.J. Herzog, D.K. Armstrong, M.F. Brady, et al., Ovarian cancer clinical trial endpoints: society of Gynecologic Oncology white paper, Gynecol. Oncol. 132 (2014) 8-17.

[30] European Medicines Agency, Appendix 1 to the Guideline on the Evaluation of Anticancer Medicinal Products in man(London, UK) 2012.

[31] P. Witteveen, Final overall survival (OS) results from AURELIA, an open-labe randomised phase III trial of chemotherapy (CT) with or without bevacizumab (BEV) for platinum resistant recurrent ovarian cancer (OC) [abstract], Eur. J. Cancer 49 (2013) (LBA 5).

[32] J.S. Ferriss, J.J. Java, M.A. Bookman, et al., Ascites predicts treatment benefit of bevacizumab in front-line therapy of advanced epithelial ovarian, fallopian tube and peritoneal cancers: an NRG oncology/GOG study, Gynecol. Oncol. 139 (2015) $17-22$.

[33] E. Smolle, V. Taucher, J. Haybaeck, Malignant ascites in ovarian cancer and the role of targeted therapeutics, Anticancer Res. 34 (2014) 1553-1561.

[34] L.E. Puls, T. Duniho, J.E. Hunter, R. Kryscio, D. Blackhurst, H. Gallion, The prognostic implication of ascites in advanced-stage ovarian cancer, Gynecol. Oncol. 61 (1996) $109-112$.

[35] W.H. Gotlieb, F. Amant, S. Advani, et al., Intravenous aflibercept for treatment of recurrent symptomatic malignant ascites in patients with advanced ovarian cancer: a phase 2, randomised, double-blind, placebo-controlled study, Lancet Oncol. 13 (2012) 154-162.

[36] N. Colombo, G. Mangili, S. Mammoliti, et al., A phase II study of aflibercept in patients with advanced epithelial ovarian cancer and symptomatic malignant ascites, Gynecol. Oncol. 125 (2012) 42-47.

[37] B.J. Monk, L. Minion, S. Lambrechts, I.B. Vergote, N. Devoogdt, B.Y. Karlan, Incidence and management of edema associated with trebananib (AMG 386), Gynecol. Oncol. 130 (2013) 636-641. 